\title{
Translation-invariant Gibbs measures for a model with logarithmic potential on a Cayley tree
}

\author{
Yu. Kh. Eshkabilov ${ }^{1}$, Sh. P. Bobonazarov ${ }^{2}$, R. I. Teshaboev ${ }^{3}$ \\ ${ }^{1}$ National University of Uzbekistan, Tashkent, Uzbekistan \\ ${ }^{2}$ Tashkent Institute of Irrigation and Melioration, Tashkent, Uzbekistan \\ ${ }^{3}$ Termez State University, Termez, Uzbekistan \\ yusup62@mail.ru, bshp@mail.ru, teshaboyev_1990@mail.ru
}

\section{DOI 10.17586/2220-8054-2016-7-5-893-899}

\begin{abstract}
In this paper, we consider a model with logarithmical potential and with the set $[0,1]$ of spin values, on a Cayley tree $\Gamma^{k}$ of the order $k$. In the case $k=2,3$, we shall prove that, there is a unique translation-invariant splitting Gibbs measure for this model. For the case $k=4$, we show that there are three translation-invariant Gibbs measures for this model.
\end{abstract}

Keywords: Cayley tree, configuration, translation-invariant Gibbs measure, fixed point, nonlinear operator.

Received: 15 April 2016. Revised: 25 May 2016.

\section{Introduction}

One of the central problems in the theory of Gibbs measures is to describe infinite-volume (or limiting) Gibbs measures corresponding to a Hamiltonian. The existence of such measures for a wide class of Hamiltonians was established in the ground-breaking work of Dobrushin. However, complete analysis of a set of limiting GMs for a specific Hamiltonian is quite often a difficult problem.

In $[1,2,6,9-11,14-16]$ for several models on Cayley tree $\Gamma^{k}$ with the order $k$, using the Markov random field theory, Gibbs measures are described. These papers are devoted to models with a finite set of spin values. In [8], the Potts model with a countable set of spin values on a Cayley tree $\Gamma^{k}$ is considered and it was shown that the set of translation-invariant splitting Gibbs measures of the model contains at most one point, independently on parameters of the Potts model with countable set of spin values on the Cayley tree. This is a crucial difference from the models with a finite set of spin values, since those may have more than one translation-invariant Gibbs measure.

In [12], a Hamiltonian with an uncountable set (a set with continuum cardinality) of spin values (with the set $[0,1]$ of spin values) on a Cayley tree $\Gamma^{k}$ is considered and it was shown that: the existence translation-invariant splitting Gibbs measure of the Hamiltonian is equivalent to the existence a positive fixed point of some nonlinear integral operator. For $k=1$, the model with the continuous potential function was shown to have a unique translation-invariant splitting Gibbs measure. In the case $k \geq 2$, some models which have the unique splitting Gibbs measure were constructed. In the paper [4], sufficient conditions were found for the potential function of the model on a Cayley tree $\Gamma^{k}$ with an uncountable set of spin values under which the model had unique translation-invariant splitting Gibbs measure. In [3,5], several models were constructed, of which these models had at least two translational-invariant Gibbs measures, i.e the existence of phase transition for some models on a Cayley tree $\Gamma^{k}(k \geq 2)$ was proven.

This paper is a continuation of previous investigations [3-5,12]. We shall construct model with a logarithmic potential on a Cayley tree $\Gamma^{k}$. We reduced the studying of translation-invariant Gibbs measures to a description of the fixed points for some nonlinear operator on $\mathbb{R}^{2}$. In the case $k=2,3$, we shall prove that, for the Hamiltonian on a Cayley tree $\Gamma^{k}$ with logarithmic potential, there is a unique translation-invariant splitting Gibbs measure. In the case $k=4$, we show that, for the model on $\Gamma^{4}$ with the logarithmic potential there are three translation-invariant Gibbs measures, i.e. there is a phase transition.

\section{Preliminaries}

A Cayley tree $\Gamma^{k}=(V, L)$ of order $k \in \mathbb{N}$ is an infinite homogeneous tree, i.e., a graph without cycles, with exactly $k+1$ edges incident to each vertex. Here, $V$ is the set of vertices and $L$ that of edges (arcs).

Consider models where the spin takes values in the set $[0,1]$, and is assigned to the vertices of the tree. For $A \subset V$, a configuration $\sigma_{A}$ on $A$ is an arbitrary function $\sigma_{A}: A \rightarrow[0,1]$. We denote $\Omega_{A}=[0,1]^{A}$ the set of all 
configurations on $A$. A configuration $\sigma$ on $V$ is then defined as a function $x \in V \mapsto \sigma(x) \in[0,1]$; the set of all configurations is $[0,1]^{V}$. The Hamiltonian of the model is :

$$
H(\sigma)=-J \sum_{\langle x, y\rangle \in L} \xi_{\sigma(x), \sigma(y)}, \quad \sigma \in \Omega_{V},
$$

where $J \in \mathbb{R} \backslash\{0\}$ and $\xi:(u, v) \in[0,1]^{2} \rightarrow \xi_{u v} \in \mathbb{R}$ is a given bounded, measurable function. As usual, $\langle x, y\rangle$ represents the nearest neighbor vertices.

Let $\lambda$ be the Lebesgue measure on $[0,1]$. On the set of all configurations on $A$, the a priori measure $\lambda_{A}$ is introduced as the $|A|$ fold product of the measure $\lambda$. Here and subsequently, $|A|$ denotes the cardinality of $A$. Below, $W_{m}$ represents a 'sphere' and $V_{m}$ for a 'ball' on the tree, of radius $m=1,2, \ldots$, centered at a fixed vertex $x^{0}$ (an origin):

$$
W_{m}=\left\{x \in V: d\left(x, x^{0}\right)=m\right\}, \quad V_{m}=\left\{x \in V: d\left(x, x^{0}\right) \leq m\right\}
$$

and

$$
L_{m}=\left\{\langle x, y\rangle \in L: x, y \in V_{m}\right\} .
$$

Here, distance $d(x, y), x, y \in V$, is the length of (i.e. the number of edges in) the shortest path connecting $x$ with $y . \Omega_{V_{n}}$ is the set of configurations in $V_{n}$ (and $\Omega_{W_{n}}$ that in $W_{n}$; see below). Furthermore, $\left.\sigma\right|_{V_{n}}$ and $\left.\omega\right|_{W_{n+1}}$ denote the restrictions of configurations $\sigma, \omega \in \Omega$ to $V_{n}$ and $W_{n+1}$, respectively. Next, $\sigma_{n}: x \in V_{n} \mapsto \sigma_{n}(x)$ is a configuration in $V_{n}$. For each $\sigma_{n} \in \Omega_{V_{n}}$, we define:

$$
H\left(\sigma_{n}\right)=-J \sum_{\langle x, y\rangle \in L_{n}} \xi_{\sigma_{n}(x), \sigma_{n}(y)} .
$$

We write $x<y$ if the path from $x^{0}$ to $y$ goes through $x$. Call vertex $y$ a direct successor of $x$ if $y>x$ and $x, y$ are nearest neighbors. We denote by $S(x)$ the set of direct successors of $x$. We observe that any vertex $x \neq x^{0}$ has $k$ direct successors and $x^{0}$ has $k+1$.

Let $h: x \in V \mapsto h_{x}=\left(h_{t, x}, t \in[0,1]\right) \in R^{[0,1]}$ be mapping of $x \in V \backslash\left\{x^{0}\right\}$. Given $n=1,2, \ldots$, consider the probability distribution $\mu^{(n)}$ on $\Omega_{V_{n}}$ defined by

$$
\mu^{(n)}\left(\sigma_{n}\right)=Z_{n}^{-1} \exp \left(-\beta H\left(\sigma_{n}\right)+\sum_{x \in W_{n}} h_{\sigma(x), x}\right),
$$

where $\beta=\frac{1}{T}, T>0$ is temperature. Here, as before, $\sigma_{n}: x \in V_{n} \mapsto \sigma(x)$ and $Z_{n}$ is the corresponding partition function:

$$
Z_{n}=\int_{\Omega_{V_{n}}} \exp \left(-\beta H\left(\widetilde{\sigma}_{n}\right)+\sum_{x \in W_{n}} h_{\widetilde{\sigma}(x), x}\right) \lambda_{V_{n}}\left(d \widetilde{\sigma}_{n}\right) .
$$

The probability distributions $\mu^{(n)}$ are compatible [12] if for any $n \geq 1$ and $\sigma_{n-1} \in \Omega_{V_{n-1}}$ :

$$
\int_{\Omega_{W_{n}}} \mu^{(n)}\left(\sigma_{n-1} \vee \omega_{n}\right) \lambda_{W_{n}}\left(d\left(\omega_{n}\right)\right)=\mu^{(n-1)}\left(\sigma_{n-1}\right) .
$$

Here, $\sigma_{n-1} \vee \omega_{n} \in \Omega_{V_{n}}$ is the concatenation of $\sigma_{n-1}$ and $\omega_{n}$. In this case, there exists [12] a unique measure $\mu$ on $\Omega_{V}$ such that, for any $n$ and $\sigma_{n} \in \Omega_{V_{n}}, \mu\left(\left\{\left.\sigma\right|_{V_{n}}=\sigma_{n}\right\}\right)=\mu^{(n)}\left(\sigma_{n}\right)$.

The measure $\mu$ is called the splitting Gibbs measure corresponding to Hamiltonian (2.1) and function $x \mapsto h_{x}$, $x \neq x^{0}$.

Proposition 2.1. [12] The probability distributions $\mu^{(n)}\left(\sigma_{n}\right), n=1,2, \ldots$, in (2.2) are compatible iff for any $x \in V \backslash\left\{x^{0}\right\}$ the following equality holds:

$$
f(t, x)=\prod_{y \in S(x)} \frac{\int_{0}^{1} \exp \left(J \beta \xi_{t u}\right) f(u, y) d u}{\int_{0}^{1} \exp \left(J \beta \xi_{0 u}\right) f(u, y) d u} .
$$

Here, and below $f(t, x)=\exp \left(h_{t, x}-h_{0, x}\right), t \in[0,1]$ and $d u=\lambda(d u)$ is the Lebesgue measure. 
From Proposition 2.1, it follows that for any $h=\left\{h_{x} \in R^{[0,1]}, \quad x \in V\right\}$ satisfying (2.5) there exists a unique Gibbs measure $\mu$ and vice versa. However, the analysis of solutions to (2.5) is not easy. Let $\xi_{t u}$ be a continuous function. We put

$$
C^{+}[0,1]=\{f \in C[0,1]: f(x) \geq 0\}, \quad C_{0}^{+}[0,1]=C^{+}[0,1] \backslash\{\theta \equiv 0\} .
$$

We define the operator $R_{k}: C_{0}^{+}[0,1] \rightarrow C_{0}^{+}[0,1]$ by

$$
\left(R_{k} f\right)(t)=\left(\frac{\int_{0}^{1} K(t, u) f(u) d u}{\int_{0}^{1} K(0, u) f(u) d u}\right)^{k}, k \in \mathbb{N}
$$

where $K(t, u)=\exp \left(J \beta \xi_{t u}\right), f(t)>0, t, u \in[0,1]$.

We will solve the equation (2.5) in the class of translational-invariant functions $f(t, x)$, i.e $f(t, x)=f(t)$ for any $x \in V$. For such functions, equation (2.5) can be written as:

$$
R_{k}(f)(t)=f(t) .
$$

Note that equation (2.6) is not linear for any $k \in \mathbb{N}$. For every $k \in \mathbb{N}$ we consider Hammerstein's integral operator $H_{k}$ acting in the cone $C^{+}[0,1]$ as

$$
\left(H_{k} f\right)(t)=\int_{0}^{1} K(t, u) f^{k}(u) d u, \quad k \in \mathbb{N} .
$$

We denote

$$
\mathcal{M}_{0}=\left\{f \in C^{+}[0,1]: \quad f(0)=1\right\} .
$$

Lemma 2.2. [4] Let $k \geq 2$. The equation

$$
R_{k} f=f, \quad f \in C_{0}^{+}[0,1]
$$

has a nontrivial positive solution iff the Hammerstein's equation

$$
H_{k} f=\lambda f, \quad f \in C^{+}[0,1]
$$

has a positive solution in $\mathcal{M}_{0}$ for some $\lambda>0$.

Let $k \geq 2$. Then, we can easily verify that: if the number $\lambda_{0}>0$ is eigenvalue of the operator $H_{k}$, then an arbitrary positive number is an eigenvalue of the operator $H_{k}$ (see [4]). Consequently, we obtain:

Lemma 2.3. Let $k \geq 2$. The equation (2.7) has a nontrivial positive solution iff the Hammerstein's operator $H_{k}$ has a nontrivial positive fixed point. Moreover, the number of nontrivial positive fixed points of the operator $R_{k}$ is equal to the number of nontrivial positive fixed points of the Hammerstein's operator $H_{k}$.

Note, that if there is more than one nontrivial positive fixed point for the the Hammerstein's operator, $H_{k}$, then there is more than one translation-invariant Gibbs measure for the model (2.1) corresponding to these fixed points. We say that a phase transition occurs for the model (2.1), if the Hammerstein's operator $H_{k}$ has more than one nontrivial positive fixed point. The number of the fixed points depends on the parameters of the model (2.1) and the order of Cayley tree $\Gamma^{k}$.

\section{A model on Cayley tree with logarithmic potential}

We consider Hamiltonian $H$ on the Cayley tree $\Gamma^{k}$ by rule:

$$
H(\sigma)=-\sum_{\langle x, y\rangle \in L} \frac{\ln \left(1+4 \theta\left(\sigma(x)-\frac{1}{2}\right)\left(\sigma(y)-\frac{1}{2}\right)\right)}{\beta}, \sigma \in \Omega_{V},
$$

where $\theta$ is a coupling constant and $0<\theta<1$, i.e. in the (2.1) function of potential is defined by the formula:

$$
\xi_{t, u}=\frac{\ln \left(1+4 \theta\left(t-\frac{1}{2}\right)\left(u-\frac{1}{2}\right)\right)}{J \beta} .
$$

The main aim of this paper is to study translation-invariant Gibbs measures for model (3.1) on the Cayley tree $\Gamma^{k}$. We define Hammerstein's operator $H_{k}$ on $C[0,1]$ by the equality:

$$
\left(H_{k} f\right)(t)=\int_{0}^{1}\left(1+4 \theta\left(t-\frac{1}{2}\right)\left(u-\frac{1}{2}\right)\right) f^{k}(u) d u .
$$


We set:

$$
k_{1}= \begin{cases}k, & \text { if } k \text { is even } \\ k-1, & \text { if } k \text { is odd }\end{cases}
$$

and

$$
k_{2}= \begin{cases}k, & \text { if } k \text { is odd } \\ k-1, & \text { if } k \text { is even }\end{cases}
$$

We define operator $P$ on $\mathbb{R}^{2}$ by the rule:

$$
P(x, y) \rightarrow\left(x^{\prime}, y^{\prime}\right)
$$

where

$$
\begin{gathered}
x^{\prime}=\sum_{j=0}^{k_{1} / 2} \frac{(2 \theta)^{2 j}}{2 j+1} A_{k}^{2 j} x^{k-2 j} y^{2 j}, \\
y^{\prime}=\sum_{j=1}^{\left(k_{2}+1\right) / 2} \frac{(2 \theta)^{2 j-1}}{2(2 j+1)} A_{k}^{2 j-1} x^{k-2 j+1} y^{2 j-1} .
\end{gathered}
$$

Here

$$
A_{n}^{m}=\frac{n !}{m !(n-m) !}
$$

Lemma 3.1. Let $k \geq 2$. The Hammerstein's operator $H_{k}$ (3.2) has a nontrivial positive fixed point iff the operator $P$ has a fixed point $\left(x_{0}, y_{0}\right)$, such that $x_{0}>0$ and $f_{0}(t)=x_{0}+4 \theta y_{0}\left(t-\frac{1}{2}\right)>0$ for all $t \in[0,1]$, moreover the function $f_{0}(t)=x_{0}+4 \theta y_{0}\left(t-\frac{1}{2}\right)$ is a positive fixed point of the Hammerstein's operator $H_{k}$.

Proof. Necessity. We set:

$$
c_{1}=\int_{0}^{1} f^{k}(u) d u
$$

and

$$
c_{2}=\int_{0}^{1}\left(u-\frac{1}{2}\right) f^{k}(u) d u .
$$

It is clear, that $c_{1}>0$. Let the Hammerstein's operator $H_{k}$ (3.2) has a positive fixed point $f(t)$. Then, for the function $f(t)$, the equality:

is holds.

$$
f(t)=c_{1}+4 \theta c_{2}\left(t-\frac{1}{2}\right)
$$

Consequently, for the parameter $c_{1}$, from the equality (3.3), we have:

$$
\begin{aligned}
c_{1}=\int_{0}^{1}\left(c_{1}+4 \theta c_{2}\left(u-\frac{1}{2}\right)\right)^{k} d u=\sum_{j=0}^{k} A_{k}^{j} c_{1}^{k-j}\left(4 \theta c_{2}\right)^{j} \int_{0}^{1}\left(u-\frac{1}{2}\right)^{j} d u= \\
=\sum_{j=0}^{k} A_{k}^{j} c_{1}^{k-j}\left(4 \theta c_{2}\right)^{j} \int_{-1 / 2}^{1 / 2} u^{j} d u=\sum_{j=0}^{k_{1} / 2} \frac{(2 \theta)^{2 j}}{2 j+1} A_{k}^{2 j} c_{1}^{k-2 j} c_{2}^{2 j} .
\end{aligned}
$$

Analogously, for the parameter $c_{2}$, by equality (3.4), we get:

$$
\begin{aligned}
c_{2}=\int_{0}^{1}\left(u-\frac{1}{2}\right)\left(c_{1}+4 \theta c_{2}\left(u-\frac{1}{2}\right)\right)^{k} d u=\sum_{j=0}^{k} A_{k}^{j} c_{1}^{k-j}\left(4 \theta c_{2}\right)^{j} \int_{0}^{1}\left(u-\frac{1}{2}\right)^{j+1} d u \\
=\sum_{j=0}^{k} A_{k}^{j} c_{1}^{k-j}\left(4 \theta c_{2}\right)^{j} \int_{-1 / 2}^{1 / 2} u^{j+1} d u=\sum_{j=1}^{\left(k_{2}+1\right) / 2} \frac{(2 \theta)^{2 j-1}}{2 j+1} A_{k}^{2 j-1} c_{1}^{k-2 j+1} c_{2}^{2 j-1} .
\end{aligned}
$$


Therefore, the point $\left(c_{1}, c_{2}\right)$ is a fixed point of the operator $P$.

Sufficiency. We assume that $x_{0}>0$ and the point $\left(x_{0}, y_{0}\right)$ is a fixed point of the operator $P$, i.e. the following equalities for numbers $x_{0}$ and $y_{0}$ numbers are satisfied:

$$
\sum_{j=0}^{k_{1} / 2} \frac{(2 \theta)^{2 j}}{2 j+1} A_{k}^{2 j} x_{0}^{k-2 j} y_{0}^{2 j}=x_{0}, \quad \sum_{j=1}^{\left(k_{2}+1\right) / 2} \frac{(2 \theta)^{2 j-1}}{2(2 j+1)} A_{k}^{2 j-1} x_{0}^{k-2 j+1} y_{0}^{2 j-1}=y_{0} .
$$

We can simply prove that the function $f_{0}(t)=x_{0}+4 \theta y_{0}\left(t-\frac{1}{2}\right)$ is a fixed point of the Hammerstein's operator $H_{k}$, i.e. $H_{k} f_{0}=f_{0}$. This completes the proof.

Proposition 3.2. For each $k \in \mathbb{N}$, the function $f_{0}(t) \equiv 1$ is a fixed point of the Hammerstein's operator $H_{k}$.

Proof. One can clearly see that:

$$
\left(H_{k}\right) f_{0}(t)=\int_{0}^{1}\left(1+4 \theta\left(t-\frac{1}{2}\right)\left(u-\frac{1}{2}\right)\right) d u==\int_{0}^{1} d u+4 \theta\left(t-\frac{1}{2}\right) \int_{-1 / 2}^{1 / 2} u d u=1=f_{0}(t) .
$$

\section{Uniqueness of translation-invariant Gibbs measures for the model (3.1)}

In [12], a Hamiltonian with an uncountable set of spin values (with the set $[0,1]$ of spin values) on the Cayley tree $\Gamma^{k}$ was considered for a continuous potential $\xi_{t, u}$. For $k=1$, it was shown that the model (2.1) with the continuous potential function has a unique translation-invariant splitting Gibbs measure. This statement holds for the model (3.1). We study translation-invariant splitting Gibbs measure for the model (3.1) for the case $k \geq 2$.

Theorem 4.1. The model $H$ (3.1) on the Cayley tree of order two has a unique translation-invariant Gibbs measure.

Proof. Let be $k=2$. Then, the operator $P$ assumes the following simple form:

$$
P(x, y)=\left(x^{2}+\frac{4}{3} y^{2}, \frac{2}{3} \theta x y\right) .
$$

For a fixed point $(x, y)$ of the operator $P$, we have the following system of algebraic equations:

$$
\left\{\begin{array}{l}
x^{2}+\frac{4}{3} y^{2}=x \\
\frac{2}{3} \theta x y=y
\end{array}\right.
$$

It follows that, the operator $P$ has a unique nontrivial fixed point $(1,0)$, as $\theta \in(0,1)$. By lemma 3.1, the Hammerstein's operator $H_{2}$ has a unique nontrivial positive fixed point $f_{0}(t) \equiv 1$. Therefore, by lemma 2.3 , the model $H$ (3.1) on the Cayley tree of order two has a unique translation-invariant Gibbs measure.

Theorem 4.2. The model $H$ (3.1) on the Cayley tree of order three has the unique translation-invariant Gibbs measure.

Proof. Let $k=3$. Then, the operator $P$ assumes the following form:

$$
P(x, y)=\left(x^{3}+4 \theta^{2} x y^{2}, \theta x^{2} y+\frac{4}{5} \theta^{3} y^{3}\right) .
$$

For a fixed point $(x, y)$ of the operator $P$, we have the following system of algebraic equations:

$$
\left\{\begin{array}{l}
x^{3}+4 \theta^{2} x y^{2}=x \\
\theta x^{2} y+\frac{4}{5} \theta^{3} y^{3}=y
\end{array}\right.
$$

It follows that, the point $(1,0)$ is a fixed point of the operator $P$. Consequently, by lemma 3.1 , the function $f_{0}(t) \equiv 1$ is a fixed point of the Hammerstein's operator $H_{3}$. Conversely, for the case $x>0, y \neq 0$, the last system of algebraic equations is equivalent to the following system of algebraic equations:

$$
\left\{\begin{array}{l}
x^{2}+4 \theta^{2} y^{2}=1 \\
\theta x^{2}+\frac{4}{5} \theta^{3} y^{2}=1
\end{array}\right.
$$


We find $x^{2}=1-4 \theta^{2} y^{2}$. Hence, for $y$, we have:

$$
\left(1-4 \theta^{2} y^{2}\right) \theta+\frac{4}{5} \theta^{3} y^{2}=1,
$$

i.e.

This is impossible, as $\theta \in(0,1)$.

$$
y^{2}=\frac{5(\theta-1)}{16 \theta^{3}} .
$$

Thus, the operator $P$ has a unique nontrivial fixed point $(1,0)$. Therefore, by lemmas 3.1 and 2.3 , the model $H$ (3.1) on the Cayley tree of order three has a unique translation-invariant Gibbs measure.

\section{A phase transition for the model (3.1)}

In this section, we consider the model (3.1) on the Cayley tree $\Gamma^{4}$. In the case $k=4$, the operator $P$ is acting on $\mathbb{R}^{2}$ by the rule:

$$
P(x, y)=\left(x^{4}+8 \theta^{2} x^{2} y^{2}+\frac{16}{5} \theta^{4} y^{4}, \quad \frac{4}{3} \theta x^{3} y+\frac{16}{5} \theta^{3} x y^{3}\right)
$$

Theorem 5.1. Let $k=4$. Then:

(a) for all $\theta \in(0,3 / 4]$ the model $H$ (3.1) on the Cayley tree $\Gamma^{k}$ has a unique translation-invariant Gibbs measure;

(b) for all $\theta \in(3 / 4,1)$ the model $H$ (3.1) on the Cayley tree $\Gamma^{k}$ has three translation-invariant Gibbs measures.

Proof. Let $k=4$. For a fixed point $(x, y)$ of the operator $P$, we have the following system of algebraic equations:

$$
\left\{\begin{array}{l}
x^{4}+8 \theta^{2} x^{2} y^{2}+\frac{16}{5} \theta^{4} y^{4}=x \\
\frac{4}{3} \theta x^{3} y+\frac{16}{5} \theta^{3} x y^{3}=y
\end{array}\right.
$$

In the case $x>0, y=0$, the above system of algebraic equations has the solution $(1,0)$. We assume that $y \neq 0$. Then, we have $x \neq 0$ and from the second equation of the last system of equations, we obtain:

$$
y^{2}=\frac{5\left(3-4 \theta x^{3}\right)}{48 \theta^{3} x} .
$$

This means that:

$$
0<x<\sqrt[3]{\frac{3}{4 \theta}}
$$

From the first equation of the system of equations, for a fixed point of the operator $P$, we obtain:

$$
\frac{16}{9} x^{6}+\frac{3 \theta-5}{3 \theta} x^{3}-\frac{5}{16 \theta^{2}}=0 .
$$

We set $z=x^{3}$. Then, $z>0$ and for the unknown variable $z$, by the equality (5.3), we have the quadratic equation:

$$
\frac{16}{9} z^{2}+\frac{3 \theta-5}{3 \theta} z-\frac{5}{16 \theta^{2}}=0
$$

One clearly sees that equation (5.4) has two roots:

where

$$
z_{1}=z_{1}(\theta)=\frac{-1+\frac{5}{3 \theta}-\sqrt{D}}{\frac{32}{9}}<0, \quad z_{2}=z_{2}(\theta)=\frac{-1+\frac{5}{3 \theta}+\sqrt{D}}{\frac{32}{9}}>0,
$$

$$
D=D(\theta)=\left(1-\frac{5}{3 \theta}\right)^{2}+\frac{20}{9 \theta^{2}} .
$$

Therefore, for $x$, by the lemma 3.1 and the inequality (5.2), we obtain $x=x_{1}=x_{1}(\theta)$, where

$$
x_{1}(\theta)=\sqrt[3]{z_{2}(\theta)}<\sqrt[3]{\frac{3}{4 \theta}} .
$$


The question then arises: does the inequality (5.2) hold for $x_{1}(\theta)$ for all values of the parameter $\theta \in(0,1)$ ? To this end, we consider the inequality $x_{1}(\theta)<\sqrt[3]{\frac{3}{4 \theta}}$. This is equivalent to the inequality:

$$
\frac{-1+\frac{5}{3 \theta}+\sqrt{D}}{\frac{32}{9}}<\sqrt[3]{\frac{3}{4 \theta}}
$$

Hence, it follows that $\sqrt{D}<1+\frac{1}{\theta}$. It means

$$
\left(1-\frac{5}{3 \theta}\right)^{2}+\frac{20}{9 \theta^{2}}<\left(1+\frac{1}{\theta}\right)^{2}
$$

From the last inequality, we get $\theta>\frac{3}{4}$. Thus, for the case $\frac{3}{4}<\theta<1$, by equality (5.1), the operator $P$ has three fixed points:

where

$$
(1,0), \quad\left(x_{1}(\theta), y_{1}(\theta)\right), \quad\left(x_{1}(\theta),-y_{1}(\theta)\right),
$$

$$
y_{1}(\theta)=\frac{1}{4 \theta} \sqrt{\frac{5\left(3-4 \theta x_{1}^{3}(\theta)\right)}{3 \theta x_{1}(\theta)}}>0 .
$$

We note that if $0<\theta \leq \frac{3}{4}$, then the operator $P$ has a unique fixed point: $(1,0)$. Consequently, by lemmas 2.3 and 3.1, for all $\theta \in\left(0, \frac{3}{4}\right]$ the model $H$ (3.1) on the Cayley tree $\Gamma^{4}$ has a unique translation-invariant Gibbs measure. In the case $\frac{3}{4}<\theta<1$ by the lemma 3.1, the Hammerstein's operator $H_{4}$ has three positive fixed points:

$$
f_{0}(t) \equiv 1, \quad f_{1}(t)=x_{1}(\theta)+4 \theta y_{1}(\theta)\left(t-\frac{1}{2}\right), \quad f_{2}(t)=x_{1}(\theta)-4 \theta y_{1}(\theta)\left(t-\frac{1}{2}\right) .
$$

Because $f_{i}(t)>0$ for all $t \in[0,1]$, where $i=1,2$. Therefore, By lemma 2.3 for all $\theta \in(3 / 4,1)$ the model $H$ (3.1) on the Cayley tree $\Gamma^{4}$ has three translation-invariant Gibbs measures. This completes the proof.

Finally we note that in the case $\theta \in\left(\frac{3}{4}, 1\right)$ for the model $H(3.1)$ on the Cayley tree $\Gamma^{4}$ there is a phase transition.

\section{References}

[1] Bleher P.M., Ganikhodjaev N.N. On pure phases of the Ising model on the Bethe lattice. Theor. Probab. Appl., 1990, 35, P. $216-227$.

[2] Bleher P.M., Ruiz J., Zagrebnov V.A. On the purity of the limiting Gibbs state for the Ising model on the Bethe lattice. Journ. Statist. Phys., 1995, 79, P. 473-482.

[3] Eshkabilov Yu.Kh., Haydarov F.H., Rozikov U.A. Non-uniqueness of Gibbs Measure for Models with Uncountable Set of Spin Values on a Cayley Tree. J. Stat. Phys., 2012, 147, P. 779-794.

[4] Eshkabilov Yu.Kh, Haydarov F.H., Rozikov U.A. Uniqueness of Gibbs Measure for Models With Uncountable Set of Spin Values on a Cayley Tree. Math. Phys. Anal. Geom., 2013, 16, P. 1-17.

[5] Eshkabilov Yu.Kh., Rozikov U.A., Botirov G.I. Phase Transitions for a Model with Uncountable Set of Spin Values on a Cayley Tree. Lobachevskii Journal of Mathematics, 2013, 34 (3), P. 256-263.

[6] Ganikhodjaev N.N. On pure phases of the ferromagnet Potts with three states on the Bethe lattice of order two. Theor. Math. Phys., 1990, 85, P. 163-175.

[7] Ganikhodjaev N.N., Rozikov U.A. Description of periodic extreme Gibbs measures of some lattice model on the Cayley tree. Theor. and Math. Phys., 1997, 111, P. 480-486.

[8] Ganikhodjaev N.N., Rozikov U.A. The Potts model with countable set of spin values on a Cayley Tree. Letters Math. Phys., 2006, 75, P. 99-109.

[9] Ganikhodjaev N.N., Rozikov U.A. On Ising model with four competing interactions on Cayley tree. Math. Phys. Anal. Geom., 2009, 12, P. 141-156.

[10] Preston C. Gibbs states on countable sets. Cambridge University Press, London, 1974.

[11] Rozikov U.A. Partition structures of the Cayley tree and applications for describing periodic Gibbs distributions. Theor. and Math. Phys., 1997, 112, P. 929-933.

[12] Rozikov U.A., Eshkabilov Yu.Kh. On models with uncountable set of spin values on a Cayley tree: Integral equations. Math. Phys. Anal. Geom., 2010, 13, P. 275-286.

[13] Sinai Ya.G. Theory of phase transitions: Rigorous Results. Pergamon, Oxford, 1982.

[14] Spitzer F. Markov random fields on an infinite tree. Ann. Prob., 1975, 3, P. 387-398.

[15] Suhov Y.M., Rozikov U.A. A hard-core model on a Cayley tree: an example of a loss network. Queueing Syst., 2004, 46, P. 197-212.

[16] Zachary S. Countable state space Markov random fields and Markov chains on trees. Ann. Prob., 1983, 11, P. 894-903. 